\title{
Serum Biomarker Status with a Distinctive Pattern in Prognosis of Gastroenteropancreatic Neuroendocrine Carcinoma
}

\author{
Jianwei Zhang ${ }^{\text {a }}$ Yanshuo Cao $^{\text {a }}$ Panpan Zhang ${ }^{\text {a }}$ Xiaotian Zhang ${ }^{\text {a }}$ Jian Li ${ }^{\mathrm{a}}$ \\ Jun Zhou ${ }^{\mathrm{a}} \quad$ Xicheng Wang $^{\mathrm{a}}$ Zhi Peng ${ }^{\mathrm{a}}$ YuSun ${ }^{\mathrm{b}} \quad$ Jie Li ${ }^{\mathrm{a}}$ Lin Shen $^{\mathrm{a}}$ Ming Lu ${ }^{\mathrm{a}}$ \\ aPeking University Cancer Hospital and Institute, Department of Gastrointestinal Oncology, Key Laboratory of \\ Carcinogenesis and Translational Research (Ministry of Education, Beijing, China), Beijing, China; ${ }^{b}$ Peking University \\ Cancer Hospital and Institute, Department of Pathology, Key Laboratory of Carcinogenesis and Translational \\ Research (Ministry of Education), Beijing, China
}

\section{Keywords}

Neuron-specific enolase · Carcinoembryonic antigen · Carbohydrate antigen 19-9. Gastroenteropancreatic neuroendocrine carcinoma · Biomarkers · Overall survival · Subtype

\begin{abstract}
Objective: Gastroenteropancreatic neuroendocrine carcinoma (GEPNEC) is a major research focus, but the application of biomarkers to guide its prognostication and management is unsatisfying. Clinical values of conventional serum biomarkers, neuron-specific enolase (NSE), carcinoembryonic antigen (CEA), and carbohydrate antigen 19-9 (CA199) warrant scrutiny. Methods: Patients diagnosed with GEPNEC with baseline NSE, CEA, and CA199 levels provided in Peking University Cancer Hospital were retrospectively studied. Relationships between biomarkers and prognosis were investigated by the $x^{2}$ test, Kaplan-Meier analysis, and univariate and multivariate Cox regression analyses. Results: A total of 640 GEPNEC patients were enrolled. NSE, CEA, and CA199 were elevated in $59.5 \%, 28.5 \%$, and $21.3 \%$ of the population, respectively. Higher NSE had worse median overall survival (OS) (17.0 months vs. not reached, hazard ratio $=2.77$ [2.06, 3.73], $p<0.001)$, and so did patients with higher CEA and CA199. Multivariable analysis confirmed that NSE and CA199
\end{abstract}

karger@karger.com www.karger.com/nen

Karger $\frac{1}{\%}$
(C) 2021 The Author(s).

Published by S. Karger AG, Basel

This is an Open Access article licensed under the Creative Commons Attribution-NonCommercial-4.0 International License (CC BY-NC) (http://www.karger.com/Services/OpenAccessLicense), applicable to the online version of the article only. Usage and distribution for commercial purposes requires written permission. correlated with OS independently. Baseline NSE level and NSE remission predicted OS and the response of patients with first-line etoposide plus cisplatin (EP) treatment. Furthermore, we combined NSE/CEA/CA199 to segregate GEPNEC into novel subgroups, namely, adenocarcinomalike NEC (ALN), neuroendocrine-like NEC (NLN), and triplenormal NEC (TNN). The groups shared distinctive clinicopathologic features and prognosis (21.0 months vs. 17.1 months vs. not reached, $p<0.001)$. The EP regimen remained the priority treatment option in NLN/TNN, while ALN was predisposed to "adenocarcinoma-like chemotherapy." Conclusions: Elevation of NSE, CEA, or CA199 was common and independently indicates poor prognosis in GEPNEC patients. Serum biomarker-based subtypes suggest meaningful clinical implications and appropriate therapeutic approaches, illuminating promising ways to characterize the prognosis of GEPNEC.

(c) 2021 The Author(s).

Published by S. Karger AG, Basel

\section{Introduction}

With the incidence of gastroenteropancreatic neuroendocrine neoplasms soaring recently, management of these diseases has been provoking more attention. In particular, the poorly differentiated entity, gastroenteropan- 
creatic neuroendocrine carcinoma (GEPNEC), has a median survival duration of 5-37 months [1-3]. Current staging systems to characterize the prognosis of GEPNEC are complicated and impractical in some situations, especially when primary sites and pathological factors are taken into consideration. Thus, simple and practical tools to discriminate survival and indicate treatment options across heterogeneous populations are urgently required [1-4]. However, the clinical value of biomarkers in NEC has long been underestimated [5]. Recently, identifying serum biomarkers of NEC has been conducive to clinical use, which warrants further reinspection.

Circulating biomarkers are classified as either diagnostic (can be used to discriminate and diagnose disease, e.g., chromogranin A [CgA] [6]), prognostic (can forecast disease course, e.g., CgA [7], neuron-specific enolase [NSE] $[8,9]$ ), or predictive (can predict response to treatment $[10,11])$. Currently, CgA is established in many aspects of neuroendocrine tumours (NETs) in guidelines, but ideal serum prognostic/predictive biomarkers for GEPNEC have long been undetermined, especially in large-sample studies $[4,8,10-12]$.

NSE, which is instrumental in aerobic glycolysis [1315 ], is elevated in $30-50 \%$ of GEPNET [16]. NSE is considered an independent marker for neuroendocrine origin tumours, exemplified in indicating poorer survival of advanced pancreatic NET (pNET), bladder-origin NEC, GEPNET, Merkel cell carcinoma, and small cell lung cancer (SCLC) [8, 9, 13, 17-25]. In addition, carcinoembryonic antigen (CEA) and carbohydrate antigen 19-9 (CA199), which are associated with physiopathologic processes of tumorigeneses and applied in other digestive systems, have shown value in NEN [20, 26-29]. There is, however, no robust evidence of clinical implications of NSE/CEA/CA199 in GEPNEC.

We proposed to investigate the roles of serum NSE/ CEA/CA199 in patients with GEPNEC, assessing their distribution and comprehensively analysing clinicalpathologic characteristics and prognosis. Further, we attempted to divide NEC into subgroups based on these markers, anticipating uncovering more clinical traits and therapy implications, and finally determining their clinical applicability in practice.

\section{Methods}

In this study, we collected data consecutively from patients who were histologically diagnosed with GEPNEC, including pure NEC and mixed neuroendocrine non-neuroendocrine carcinoma (MiNEC) from August 1st, 2014 to August 1st, 2019 in Peking Uni- versity Cancer Hospital. All patients underwent baseline evaluations that included demographic information, clinical manifestations, pathologic characteristics, and therapeutics. The stage was evaluated by the eighth edition of the American Joint Committee on Cancer (AJCC) Tumour-Node-Metastasis system, while pathology was evaluated by the 2019 WHO classification.

Eligibility criteria included

- Patients diagnosed by experienced pathologists as poorly differentiated NEC or MiNEC.

- Baseline levels of NSE, CA199, or CEA were available.

- Patients with complete survival data.

Serum tumour biomarkers were measured at the first consultation using immunoassay, with the upper limit of normal value set as follows: serum NSE of 0-15.2 ng/mL, CA199 of 0-37 U/mL, and CEA of $0-5 \mathrm{ng} / \mathrm{mL}$. We re-assessed each biomarker after the second cycle of first-line regimens and defined biochemical remission as over $50 \%$ decrease than its baseline level. All patients were regularly followed up through outpatient clinics or phone calls. The first follow-up was performed within 3 months with subsequent follow-up cycles ranging from 6 to 12 months. The primary outcome was overall survival (OS), calculated from date of diagnosis to death by any cause or date of last follow-up. Therapy was divided into 3 categories: etoposide-platinum, irinotecan-platinum (IP), and "adenocarcinoma-like chemotherapy" (other non-EP/IP chemotherapy which focus mainly on adenocarcinoma of corresponding primary locations, namely, FOLFOX, XELOX, etc.). Response was assessed every 6 weeks for the first 6 months using Response Evaluation Criteria in Solid Tumours (RECIST) version 1.1 , according to which the objective response rate (ORR; defined as the percentage of patients with complete or partial response), and the disease control rate (DCR; defined as the percentage of patients with objective response and stable disease) or progressive disease rate were calculated.

Pearson $\chi^{2}$ tests (Fisher's test, when necessary) and one-way analysis of variance (2-sided $t$-tests, when 2 groups) were implemented to show the differences between the groups for categorical variables and continuous variables, respectively. Tumour biomarkers were assessed as categorical (normal vs. elevated) variables for associating with the OS and adjusted for confounding. Survival probabilities were revealed using the Kaplan-Meier method with log-rank test to examine the association. Univariate and multivariate analyses employing the Cox proportional hazards model identified independent factors statistically relevant to survival. All $p$ values were two-sided, and $p<0.05$ was considered significant. We utilized R version 3.5.2 (The R Foundation, Vienna, Austria) to perform these procedures.

\section{Results}

\section{Baseline Information of GEPNEC}

Among 2,048 patients recruited in our centre, $640 \mathrm{GE}-$ PNEC patients, including 463 pure NEC and 177 MiNEC, were determined to be eligible for the study (online suppl. Fig. 1; see www.karger.com/doi/10.1159/000519948 for all online suppl. material). The average age was $58.4 \pm$ 11.3 and the sex ratio was $2.64(\mathrm{M} / \mathrm{F})$. The top primary 


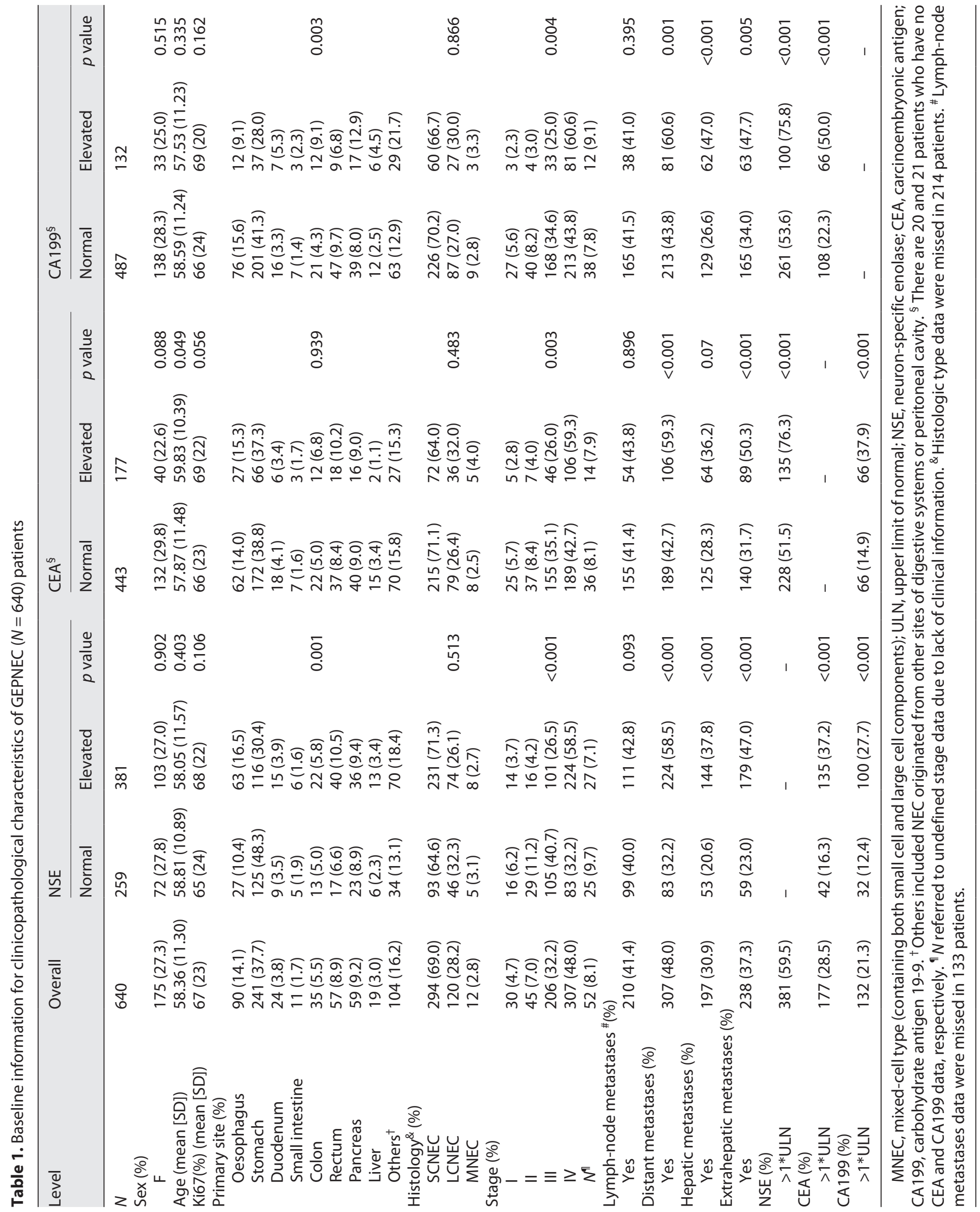



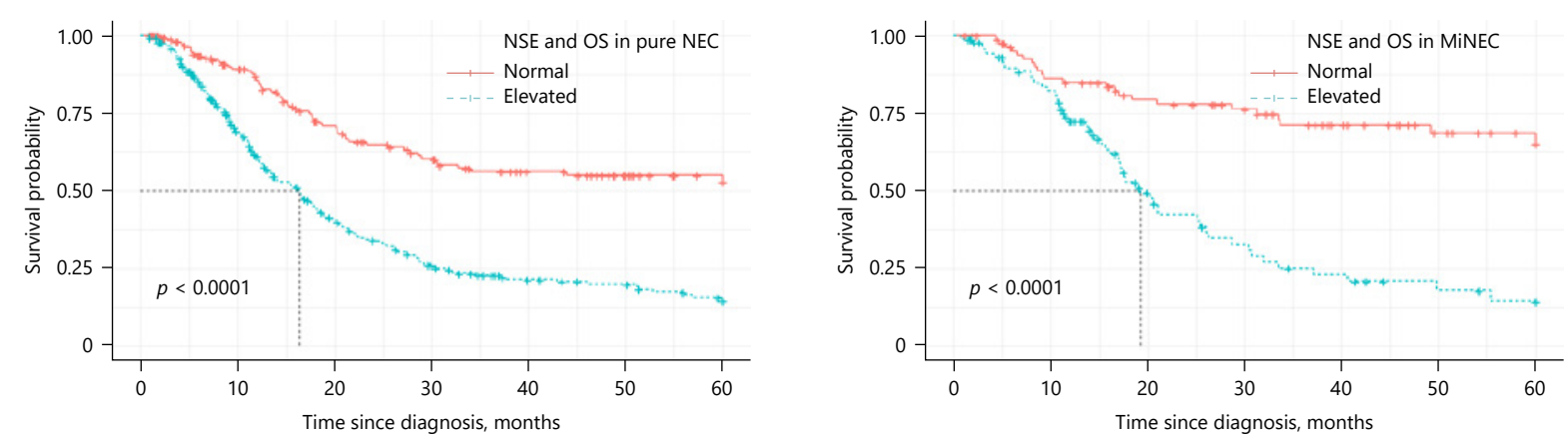

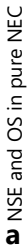
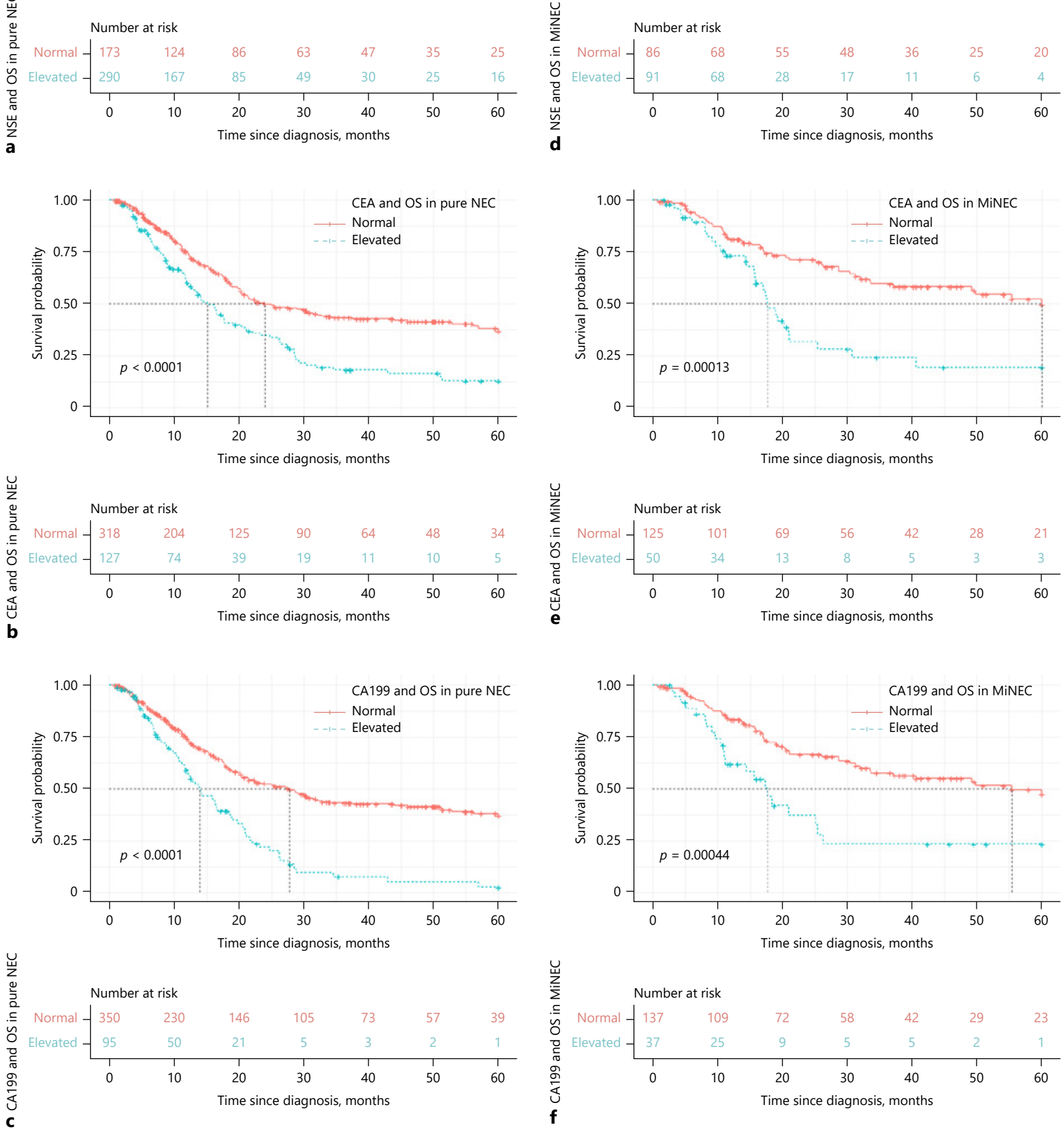

(For legend see next page.) 
site was stomach $(37.7 \%)$, followed by oesophagus (14.1\%), pancreas $(9.2 \%)$, and rectum $(8.9 \%)$. Baseline metastatic diseases were observed in $48.0 \%$ of patients. The median follow-up time was 32.8 months, and the median survival duration was 21.3 months. Elevated NSE, CEA, and CA199 had prevalence values in GEPNEC of 381 (59.5\%), 177 (28.5\%), and 132 (21.3\%), respectively. The stage and distant metastases in elevated biomarkers were all significantly different, with $p$ values $<0.05$ (Table 1). We also compared elevation of biomarkers with different anatomical sites. NSE elevation was mostly seen in patients with oesophagus and rectum NEC, while colon NEC patients had the highest CEA elevation rate, and pancreatic NEC patients had the highest CA199 elevation. Gastric NEC (gNEC), on the other hand, had relatively low proportions of NSE elevation, significantly lower than oesophagus and rectum counterparts (online suppl. Fig. 3 ). For pathologic type, we observed that only NSE in small cell GEPNEC (GEPSCNEC) patients was relatively higher than large cell GEPNEC (GEPLCNEC), though differences were not significant (online suppl. Fig. 4).

\section{Biomarkers in Prognosis}

We specifically reviewed the clinical-pathologic characteristics of subgroups, given the distinctions between pure NEC and MiNEC (online suppl. Table 1). Among 463 pure NEC patients (72.3\%), up to 291 (62.9\%) patients had elevated NSE, 127 (28.5\%) had elevated CEA, and $95(21.3 \%)$ had elevated CA199 $(p<0.001)$. All markers were linked with advanced stage (all $p<0.001)$. Elevated NSE group had higher rates of metastatic disease (59.1\% vs. $34.9 \%)$, CA199 associated with hepatic metastases (51.6\% vs. $27.6 \%)$, while CEA associated with extrahepatic metastases $(52.0 \%$ vs. $33.5 \%)$ (all $p<0.001)$. Conversely, histologic types and Ki67 did not correlate with biomarker levels. KM survival analysis revealed significant differences between elevated versus normal NSE (17.0 months vs. not reached) (Fig. 1), as well as CA199 (15.9vs. 29.0 months) and CEA (16.6 vs. 25.5 months) (all $p<0.0001)$. MiNEC $(n=177,27.7 \%)$ was also investigated (online suppl. Table 1). We found that $85.5 \%$ of mixed components were adenocarcinoma, while the rest were squamous carcinoma (10.5\%) and mixed non-neu-

Fig. 1. OS regarding the biomarkers elevation in NEC: pure NEC with NSE (a), CEA (b), CA199 (c) and MiNEC with NSE (d), CEA (e), CA199 (f). MiNEC, mixed neuroendocrine non-neuroendocrine carcinoma; OS, overall survival; NSE, neuron-specific enolase; CEA, carcinoembryonic antigen; CA199, carbohydrate antigen 19-9.

Serum Biomarkers in GEPNEC roendocrine components. NSE (19.3 months vs. not reached, $p<0.0001)$, CEA (17.7 months vs. $60.0, p=$ 0.00013 ), and CA199 (17.7 vs. 55.3 months, $p=0.00044$ ) levels all showed significance for OS of MiNEC (Fig. 1). Significantly increased hazard ratios of NSE $(\mathrm{HR}=2.47$ [1.56-3.9], $p=0.001$ for pure NEC and HR $=5.64$ [2.3913.3], $p=0.001$ for MiNEC) were observed in multivariate analysis, while CA199 (HR $=2.29$ [1.38-3.79], $p=$ 0.001 ) had significantly worse survival in pure NEC (Table 2). We specifically analysed histologic subgroups of GEPNEC as a reference. We found that in both GEPSCNEC and GEPLCNEC, the HRs increased in the NSEelevated group, and elevated CEA indicated a worse survival in GEPSCNEC (online suppl. Table 2).

\section{Biomarkers in Predicting Therapeutic Efficacy}

We also analysed associations between biomarkers and advanced NEC patients with first-line EP regimen. Only the baseline NSE elevation group was significantly distinguished from their counterparts in OS (10.7 months vs. not reached, $p=0.0021$ ) (Fig. 2 ). The baseline NSE elevation group was also significantly different in multivariate analysis $(\mathrm{HR}=3.4[1.44,8.03], p=0.005)$ (online suppl. Table 3 ). To some extent, biomarker remission had associations with clinical events, as CEA remission predicted a better OS (12.8 months vs. 31.8 months, $p=$ 0.077 ) (online suppl. Fig. 2), and NSE remission correlated with a better ORR $(p=0.009)$ (online suppl. Table 4).

\section{Biomarker-Based Subtypes}

To better characterize therapy response, GEPNEC was classified into 3 categories via biomarkers. We defined the NSE-elevated group as "neuroendocrine-like NEC" (NLN, $n=381$ ). "Adenocarcinoma-like NEC" (ALN, $n=63$ ) was deemed as either elevated CEA or CA199 with normal NSE; those with no elevations of all biomarkers were defined as "triple-normal NEC" (TNN, $n=194$ ). For distribution of these subtypes among all sites, NLN was most common in oesophagus and rectum, while ALN was relatively more prevalent in the intestine and TNN in the stomach. Concerning clinicalpathologic features, NLN was predisposed to advanced disease (58.8\%), compared with TNN (28.6\%) and ALN (41.3\%) (Table 3). Significant differences were observed in OS of TNN, ALN, and NLN (not reached vs. 21.0 vs. 17.1 months, $p<0.001$ ) (Fig. 3). Multivariable analysis reaffirmed that NLN achieved significantly far worse OS $(\mathrm{HR}=3.92[2.55-6], p<0.001)$ than TNN (online suppl. Table 5). This was in line with treatment response, as 
Table 2. Univariate analysis applying log-rank test and multivariate analysis estimated through Cox proportional hazards model in pure NEC and MiNEC patients

\begin{tabular}{|c|c|c|c|c|c|c|}
\hline \multirow[t]{2}{*}{ Factors } & \multicolumn{3}{|c|}{ Univariate } & \multicolumn{3}{|c|}{ Multivariate } \\
\hline & $\mathrm{HR}$ & $\mathrm{Cl} 95$ & $p$ value & $\mathrm{HR}$ & $\mathrm{Cl} 95$ & $p$ value \\
\hline \multicolumn{7}{|l|}{ Pure NEC } \\
\hline Sex (M) & 1.04 & $0.78-1.37$ & 0.802 & - & - & - \\
\hline Age & 1 & $0.99-1.01$ & 0.994 & - & - & - \\
\hline Ki67 & 0.72 & $0.38-1.37$ & 0.317 & - & - & - \\
\hline Distant metastases & 3.05 & $2.36-3.95$ & 0.001 & 2.78 & $2.02-4.28$ & 0.001 \\
\hline Lymph-node metastases & 1.74 & $1.1-2.73$ & 0.017 & 1.62 & $0.97-2.69$ & 0.063 \\
\hline Hepatic metastases & 2.41 & $1.87-3.12$ & 0.001 & 0.78 & $0.37-1.63$ & 0.505 \\
\hline NSE $>1 *$ ULN & 2.77 & $2.06-3.73$ & 0.001 & 2.47 & $1.56-3.9$ & 0.001 \\
\hline CEA $>1 *$ ULN & 1.83 & $1.4-2.38$ & 0.001 & 0.97 & $0.61-1.56$ & 0.912 \\
\hline CA199 >1*ULN & 2.33 & $1.75-3.11$ & 0.001 & 2.29 & $1.38-3.79$ & 0.001 \\
\hline \multicolumn{7}{|l|}{ MiNEC } \\
\hline $\operatorname{Sex}(M)$ & 1.03 & $1.01-1.06$ & 0.006 & 1.04 & $1-1.09$ & 0.063 \\
\hline Age & 1.64 & $0.92-2.93$ & 0.092 & 1.23 & $0.49-3.09$ & 0.657 \\
\hline Ki67 & 5.43 & $2.2-13.41$ & 0.001 & 0.85 & $0.18-3.95$ & 0.835 \\
\hline Distant metastases & 14.04 & $3.34-58.96$ & 0.001 & 11.96 & $2.49-57.41$ & 0.002 \\
\hline Lymph-node metastases & 1.42 & $0.78-2.56$ & 0.251 & - & - & - \\
\hline Hepatic metastases & 3.73 & $1.82-7.64$ & 0.001 & 1.22 & $0.56-2.64$ & 0.614 \\
\hline NSE $>1 *$ ULN & 3.92 & $2.36-6.5$ & 0.001 & 5.64 & $2.39-13.3$ & 0.001 \\
\hline CEA $>1 *$ ULN & 2.47 & $1.54-3.97$ & 0.001 & 2.08 & $0.91-4.77$ & 0.083 \\
\hline CA199 >1*ULN & 2.41 & $1.45-4$ & 0.001 & 1.79 & $0.75-4.3$ & 0.19 \\
\hline
\end{tabular}

Values are $n$ (\%). $p$ values determined with likelihood ratio test for HRs in Cox proportional hazards regression $95 \% \mathrm{Cl}$. Blank values $(-)$ are too insignificant $(p>0.1)$ in univariate analysis to be included in multivariate analysis. MiNEC, mixed neuroendocrine non-neuroendocrine carcinoma; $\mathrm{Cl}$ 95, 95\% confidence interval; ULN, upper limit of normal; NSE, neuron-specific enolase; CEA, carcinoembryonic antigen; CA199, carbohydrate antigen 19-9; HR, hazard ratio.

NLN had a higher progressive disease rate $(32.5 \%)$ than ALN (32.5\%) or TNN (34.9\%) (Table 3). We specifically illustrated that ALN had the best ORR (50\%) and DCR (75\%) to "adenocarcinoma-like chemotherapy," separating it as a superior entity for non-EP regimens. EP proved to be an optimal choice in NLN patients (DCR [67\%], ORR [35\%]) and in TNN (DCR [72\%], ORR [20\%]) (Table 4).

\section{Discussion}

Compared to comprehensive biochemical indicators in SCLC, the role of circulating tumour markers to predict and monitor outcome has not been properly assessed in extra-pulmonary NEC [30]. The traditional serum marker CgA is limited because it is hard to assess and has unsatisfying power in high-grade NEN [15]. We thus examined the predictive abilities of routinely assessed NSE/ CEA/CA199 and established them as excellent serum subtypes reflecting clinical features and treatment response of GEPNEC.

Epidemiologically, we confirmed that the prevalence of NSE-elevated patients was much higher in GEPNEC than in GEPNET, and the same was true for elevated CA199 and CEA. In general, primary sites of GEPNEC correlated with NSE levels, when oesophagus and rectal NEC were significantly higher than gNEC; the reasons why these locations had higher frequency remain undetermined but have clinical implications. CEA and CA199 mainly increased in NEC of adenocyte-enriched primary sites (colon and pancreas) and the proportions were relatively low in the oesophagus, which may reflect an adenocarcinoma-like feature in these locations. Additionally, the levels of biomarkers had no pathologic specificity.

We propose that these biomarkers are favoured in evaluating prognosis during management of GEPNEC. It is widely acknowledged that NSE reflects systemic cancer burden and manifests metastases [2]. Moreover, CEA/ CA199 can also identify advanced disease, indicating 
their value in assessing tumour burden. Higher NSE is relevant to worse progression free survival/OS in advanced pNET (RADIANT-1/RADIANT-3), IV GEPNET, and SCLC $[8,9,17-20,31]$. These results are consistent with much higher elevated CEA/CA199 proportions in metastatic SCLC $[13,32,33]$. We confirmed that NSE level was predisposed to undesirable prognosis, and the same was true for CEA and CA199. We upgraded the prognostic values of CEA/CA199. CEA was previously reported to be associated with survival of SCLC, disease progression, and post-operative/treatment monitoring [32-34]. Recent studies also revealed its capability of predicting survival for patients with gNEC and gNET [26, 35]. The mechanisms underlying CEA/CA199 and aggressiveness of NEC are undefined but are possibly due to tumour hypoxia and vessel invasion [29]. Accumulating evidence suggests that higher NSE can be applied in different histologic types, as it is independently correlated with a poorer OS in SCLC $[24,36-38]$. Nonetheless, clinical implications of NSE in LCNEC survival are disputed $[39,40]$. But we demonstrated that NSE can be extended to GEPLCNEC. We recommend applying CEA to evaluate prognosis of GEPSCNEC, and applying CA199 to evaluate prognosis of GEPLCNEC.

We suggested that tumour biomarkers were endowed with predictive meanings: baseline NSE elevation may be correlated with resistance to first-line EP regimen, while NSE remission may forecast response in GEPNEC, in accordance with its predictive strength in first-line therapy in SCLC $[26,30$, 37]. Responders to CEA in first-line therapy were more likely to experience longer OS. Although no significance was detected in CA199, it was reported to predict first-line treatment response of NEN [41-43]. Further investigations may validate these discoveries.

Traditional tumour-node-metastasis staging and histopathological categories still have flaws and are not concise enough in prognosis and management of NEC [44]. Biomarker combinations may shed light on the problem, as they were previously identified to describe survival in advanced pNET and generate a predictive modelling framework (NSE and lactate dehydrogenase) for guiding SCLC therapy [8, 17, 45]. NSE is commonly acknowledged as a major neuroendocrine-marker, and CEA/ CA199 may represent "adeno-markers," so we determined whether subtypes could be constructed based on biomarkers to delineate different NEC features. NLN (with higher neuroendocrine biomarkers) usually behaves more aggressively than ALN (with higher "adenomarkers") and TNN, manifesting higher frequency of metastases and worse survival. Thus, the proportions of

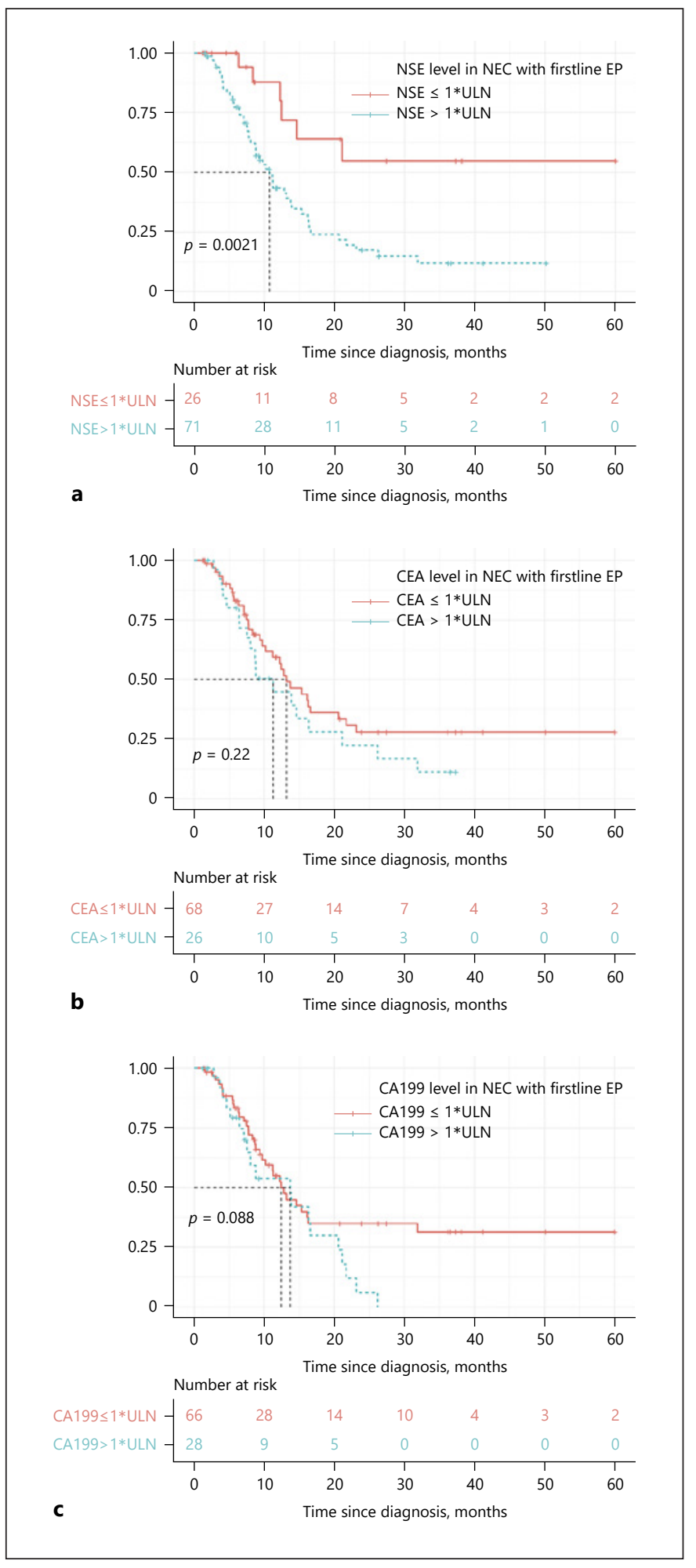

Fig. 2. Predicative values of biomarkers remission in the advanced NEC with first-line EP regimens: NSE (a), CEA (b), CA199 (c). NSE, neuron-specific enolase; CEA, carcinoembryonic antigen; CA199, carbohydrate antigen 19-9; EP, etoposide plus cisplatin; ULN, upper limit of normal value. 
Table 3. Baseline information for subtype-related clinic-pathological characteristics in NEC

\begin{tabular}{|c|c|c|c|c|c|}
\hline & Level & TNN & ALN & NLN & $p$ value \\
\hline$N$ & $638^{*}$ & 194 & 63 & 381 & \\
\hline Age (mean [SD]) & & $58.84(10.97)$ & $58.94(10.85)$ & 58.05 (11.57) & 0.676 \\
\hline \multirow[t]{2}{*}{ Sex (\%) } & $\mathrm{F}$ & $60(30.9)$ & $12(19.0)$ & $103(27.0)$ & \multirow[t]{2}{*}{0.179} \\
\hline & $M$ & $134(69.1)$ & $51(81.0)$ & $278(73.0)$ & \\
\hline \multirow[t]{6}{*}{ Site (\%) } & Oesophagus & $24(12.4)$ & $3(4.8)$ & $63(16.5)$ & \multirow{6}{*}{0.001} \\
\hline & Stomach & $94(48.5)$ & $31(49.2)$ & $116(30.4)$ & \\
\hline & Intestine & $20(10.3)$ & 10 (15.9) & $43(11.3)$ & \\
\hline & Rectum & $11(5.7)$ & $6(9.5)$ & $40(10.5)$ & \\
\hline & Pancreas & $17(8.8)$ & $6(9.5)$ & $36(9.4)$ & \\
\hline & Others & $28(14.4)$ & $7(11.1)$ & $83(21.8)$ & \\
\hline \multirow[t]{3}{*}{ Component (\%) } & Small cell & $60(60.6)$ & $23(54.8)$ & $169(67.9)$ & \multirow{3}{*}{0.433} \\
\hline & Large cell & $34(34.3)$ & $17(40.5)$ & $69(27.7)$ & \\
\hline & Mixed/unknown & $5(5.1)$ & $2(4.8)$ & $11(4.4)$ & \\
\hline Ki67\% (mean [SD]) & & $63(25)$ & $70(19)$ & $68(22)$ & 0.032 \\
\hline Lymph-node metastases (\%) & Yes & $120(65.6)$ & $39(76.5)$ & $196(77.5)$ & 0.069 \\
\hline Distant metastases (\%) & Yes & $55(28.6)$ & $26(41.3)$ & $224(58.8)$ & $<0.001$ \\
\hline Hepatic metastases (\%) & Yes & $34(17.7)$ & $17(27.0)$ & $144(37.8)$ & $<0.001$ \\
\hline Extrahepatic metastases (\%) & Yes & $38(19.8)$ & $19(30.2)$ & $179(47.0)$ & $<0.001$ \\
\hline \multirow[t]{2}{*}{ Therapy (\%) } & CT & $43(32.6)$ & $15(31.2)$ & $98(29.1)$ & \multirow{2}{*}{0.355} \\
\hline & $\mathrm{EP} / \mathrm{IP}$ & $89(67.4)$ & $33(68.8)$ & $239(70.9)$ & \\
\hline \multirow[t]{3}{*}{ Efficacy (\%) } & PR & $22(20.2)$ & $15(37.5)$ & $91(29.2)$ & \multirow{3}{*}{0.027} \\
\hline & SD & $49(45.0)$ & $12(30.0)$ & $94(30.1)$ & \\
\hline & PD & $38(34.9)$ & $13(32.5)$ & $127(40.7)$ & \\
\hline \multirow[t]{2}{*}{ DCR rate (\%) } & DCR & $71(65.1)$ & $27(67.5)$ & $185(59.3)$ & \multirow{2}{*}{0.396} \\
\hline & PD & $38(34.9)$ & $13(32.5)$ & $127(40.7)$ & \\
\hline
\end{tabular}

TNN, triple-normal NEC; ALN, adenocarinoma-like NEC; NLN, neuroendocrine-like NEC; Intestine, duodenum, small intestine, and colon; $C T$, chemotherapy on corresponding adenocarcinoma of same location; EP/IP, etoposide/irinotecan plus cisplatin regimens; $D C R$, disease control rate; $C R$, complete response; $P R$, partial response; $S D$, stable disease; PD, progression disease; NSE, neuron-specific enolase; CEA, carcinoembryonic antigen; CA199, carbohydrate antigen 19-9. * There are 2 patients without enough information for classifications (normal NSE level with lack of both CEA and CA199 information).

different-primary site NLN may account for the disparity in prognosis, for example, poorer outcome in oesophagus NEC $[3,18,46]$.

We also illustrated that contrary to the common EP regimen used in advanced NEC, ALN responded better to "adenocarcinoma-like chemotherapy," which may be conducive to shifting first-line therapy in this entity. The EP regimen was still a better option than IP in NLN and TNN, consistent with current guidelines [47]. But NLN showed an inconsistent lower DCR and shorter OS than TNN. It may be associated with innately more aggressive behaviours of NLN, and possibly related to secondary drug resistance of NLN in next-line treatment, arousing controversy on optimal sequential treatment. This association required future prospective validation on secondline therapy. Our subtypes provide novel evidence when incorporating comprehensive clinical features and are more informative in first-line therapeutic options. We recommend that our findings are utilized as alternative guidance to traditional histologic subtypes, as the subtypes may exhibit broader clinical implications when integrated with other discriminatory tools. 


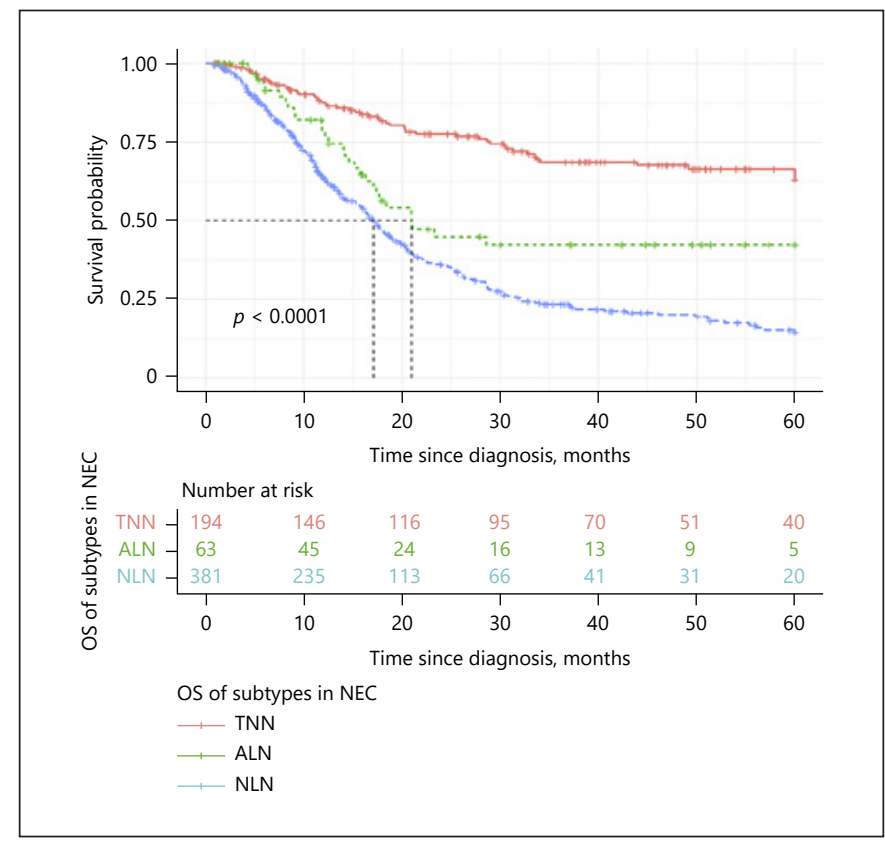

Fig. 3. OS regarding the serum-based subtypes of the NEC. There are 2 patients without enough information (normal NSE level with lack of both CEA and CA199 information). NLN, neuroendocrinelike NEC; ALN, adenocarcinoma-like NEC; TNN, triple-normal NEC; OS, overall survival; NSE, neuron-specific enolase; CEA, carcinoembryonic antigen; CA199, carbohydrate antigen 19-9.

Consequently, our study provides strong evidence of the value of biomarkers in GEPNEC, which have been ignored by previous studies. Firstly, contrary to previous reports with tremendous heterogeneity and small samples of biomarkers [48], this is a large-sample study concentrating on GEPNEC. Moreover, NSE/CA199/CEA is easily detected and had promising monitoring strength similar to traditional CgA. Novel progression of biomarkers, including circulating cell-free DNA, circulating tumour cells, microRNAs, or long non-coding RNAs, and blood transcripts (e.g., NETest) are underway for GEPNET $[49,50]$. Most discoveries are based on a small number of samples and have not reached a consistent standard [51, 52]. Our biomarkers are economical with higher performance. These classical serum biomarkers are superior in repeatability, accessibility, and intuitive interpretation compared to the high cost and immaturity of forthcoming biomarkers.

There are some limitations of this study. Firstly, it is a retrospective analysis from a single centre. To mitigate this, we carried out strict screening to enrol eligible patients with convincing data and monitor any inconsistency during the process to confirm the quality of the data. There may also be false-positive values of serum biomark-
Table 4. Therapy response to subtypes of NEC

\begin{tabular}{llllllll}
\hline Types & First-line & PR & SD & PD & $\begin{array}{l}\text { ORR, } \\
\%\end{array}$ & $\begin{array}{l}\text { DCR, } \\
\%\end{array}$ & $p$ value \\
\hline TNN & CT & 4 & 14 & 16 & 12 & 53 & \\
& EP & 11 & 28 & 15 & 20 & 72 & 0.15 \\
& IP & 2 & 3 & 6 & 18 & 45 & \\
\hline ALN & CT & 6 & 3 & 3 & 50 & 75 & \\
& EP & 3 & 6 & 7 & 19 & 56 & 0.26 \\
& IP & 3 & 3 & 5 & 27 & 55 & \\
\hline \multirow{2}{*}{ LLN } & CT & 15 & 25 & 43 & 18 & 48 & \\
& EP & 47 & 44 & 44 & 35 & 67 & $0.0073^{*}$ \\
& IP & 26 & 20 & 31 & 34 & 60 & \\
& & & & & & &
\end{tabular}

TNN, triple-normal NEC; ALN, adenocarinoma-like NEC; NLN, neuroendocrine-like NEC; EP, etoposide plus cisplatin; IP, irinotecan plus cisplatin; CT, " adenocarcinoma-like therapy" on adenocarcinoma of same locations; $\mathrm{DCR}$, disease control rate; $\mathrm{PR}$, partial response; SD, stable disease; $\mathrm{PD}$, progression disease; ORR, objective response rate. ${ }^{*} p$ value $<0.05$ as significance.

ers (especially in CEA/CA199), which is led by confounding factors, such as tobacco use, diabetes, and inflammatory conditions. Thus, there are still no optimized cut-off values. Inconsistency of the reference range of the utilized assay in different studies also hampers interpretation. We employed Cox regression models to adjust all available parameters to lower the biases. In addition, a small number of patients were pre-treated before referral to our institute or their treatment had lapsed. This accounted for missing baseline values. Various pre-referral or post-progression treatment modalities might expand heterogeneity, so we strictly enrolled and analysed 3 major regimens. Despite the day-to-day variation of all serum biomarkers, combining them with radiographic tools in evaluating treatment response may be more applicable and worth exploring.

In conclusion, our cohort allowed us to identify meaningful biomarkers (NSE/CEA/CA199) with respect to their predictive values in GEPNEC. We demonstrated that elevation of NSE, CEA, and CA199 had poorer survival. NSE was affirmed as significant (both pure NEC and MiNEC) and CA199 in NEC by multivariable analysis. NSE independently correlated with response to firstline therapy in advanced GEPNEC. We recapitulated serum biomarker pattern-based subgroups of NEC, revealing valid clinical characteristics and prognosis. Notably, the distinctive entity ALN was recommended for adenocarcinoma-like chemotherapy. Further prospective and randomized studies are warranted to validate the clinical utility of traditional tumour biomarkers in GEPNEC. 


\section{Acknowledgments}

The authors thank their colleagues and all the subjects who participated in the study for their contributions.

\section{Statement of Ethics}

We declare that the research is conducted ethically in accordance with the World Medical Association Declaration of Helsinki. We state that all subjects have given their written informed consent and that the study protocol was approved by the Ethics Committee of the Peking University School of Oncology with a reference number of 2017YJZ36.

\section{Conflict of Interest Statement}

We have read and understood the policy on disclosing conflicts of interest and have no conflicts of interest to declare.

\section{Funding Sources}

This work was supported by the third round of public welfare development and reform pilot projects of Beijing Municipal Medical Research Institutes (Beijing Medical Research Institute, 2019-1).

\section{Author Contributions}

Ming Lu, Jie Li, and Lin Shen made substantial contributions to conception and design. Ming Lu, Yu Sun, Jian Li, Jun Zhou, Xicheng Wang, and Zhi Peng helped acquire the data. Jianwei Zhang drafted the article and analysed and interpreted the data. Ming Lu, Xiaotian Zhang, Yanshuo Cao, and Panpan Zhang participated in drafting the article and revising it critically for important intellectual content.

\section{Data Availability Statement}

All data generated or analysed during this study are included in this article and its supplementary material files. Further enquiries can be directed to the corresponding author.

\section{References}

1 Yao JC, Hassan M, Phan A, Dagohoy C, Leary $\mathrm{C}$, Mares JE, et al. One hundred years after "Carcinoid": epidemiology of and prognostic factors for neuroendocrine tumors in 35,825 cases in the United States. J Clin Oncol. 2008 Jun 20;26(18):3063-72.

2 Walter T, Tougeron D, Baudin E, Le Malicot $\mathrm{K}$, Lecomte T, Malka D, et al. Poorly differentiated gastro-entero-pancreatic neuroendocrine carcinomas: are they really heterogeneous? insights from the FFCD-GTE national cohort. Eur J Cancer. 2017 Jul;79:158-65.

3 Dasari A, Mehta K, Byers LA, Sorbye H, Yao JC. Comparative study of lung and extrapulmonary poorly differentiated neuroendocrine carcinomas: a SEER database analysis of 162,983 cases. Cancer. 2018 Feb 15;124(4):807-15.

4 Sorbye H, Baudin E, Borbath I, Caplin M, Chen J, Cwikla JB, et al. Unmet needs in highgrade gastroenteropancreatic neuroendocrine neoplasms (WHO G3). Neuroendocrinol. 2019;108(1):54-62.

5 Modlin IM, Moss SF, Chung DC, Jensen RT, Snyderwine E. Priorities for improving the management of gastroenteropancreatic neuroendocrine tumors. J Natl Cancer Inst. 2008 Sep 17;100(18):1282-9.

6 Hofland J, Zandee WT, de Herder WW. Role of biomarker tests for diagnosis of neuroendocrine tumours. Nat Rev Endocrinol. 2018 Nov;14(11):656-69.

7 Zou J, Li Q, Kou F, Zhu Y, Lu M, Li J, et al. Prognostic value of inflammation-based markers in advanced or metastatic neuroendocrine tumours. Curr Oncol. 2019;26(1): e30-8.
8 Liu X, Zhang W, Yin W, Xiao Y, Zhou C, Hu $\mathrm{Y}$, et al. The prognostic value of the serum neuron specific enolase and lactate dehydrogenase in small cell lung cancer patients receiving first-line platinum-based chemotherapy. Medicine. 2017 Nov;96(46):e8258.

9 Yao JC, Pavel M, Lombard-Bohas C, Van Cutsem E, Voi M, Brandt U, et al. Everolimus for the treatment of advanced pancreatic neuroendocrine tumors: overall survival and circulating biomarkers from the randomized, phase III RADIANT-3 study. J Clin Oncol. 2016 Nov 10;34(32):3906-13.

10 O'Toole D, Grossman A, Gross D, Delle Fave G, Barkmanova J, O'Connor J, et al. ENETS consensus guidelines for the standards of care in neuroendocrine tumors: biochemical markers. Neuroendocrinol. 2009;90(2):194-202.

11 Oberg K, Couvelard A, Delle Fave G, Gross D, Grossman A, Jensen RT, et al. ENETS consensus guidelines for standard of care in neuroendocrine tumours: biochemical markers. Neuroendocrinol. 2017;105(3):201-11.

12 Seregni E, Ferrari L, Bajetta E, Martinetti A, Bombardieri E. Clinical significance of blood chromogranin A measurement in neuroendocrine tumours. Ann Oncol. 2001;12(Suppl 2):S69-72.

13 Harmsma M, Schutte B, Ramaekers FC. Serum markers in small cell lung cancer: opportunities for improvement. Biochim Biophys Acta. 2013 Dec;1836(2):255-72.

14 Nobels FR, Kwekkeboom DJ, Coopmans W, Schoenmakers CH, Lindemans J, De Herder WW, et al. Chromogranin A as serum marker for neuroendocrine neoplasia: comparison with neuron-specific enolase and the alphasubunit of glycoprotein hormones. J Clin Endocrinol Metab. 1997 Aug;82(8):2622-8.

15 Sansone A, Lauretta R, Vottari S, Chiefari A, Barnabei A, Romanelli F, et al. Specific and non-specific biomarkers in neuroendocrine gastroenteropancreatic tumors. Cancers. 2019 Aug 4;11(8).

16 Oberg K. Circulating biomarkers in gastroenteropancreatic neuroendocrine tumours. Endocr Relat Cancer. 2011 Oct;18(Suppl 1):S1725.

17 Yao JC, Pavel M, Phan AT, Kulke MH, Hoosen S, St Peter J, et al. Chromogranin A and neuron-specific enolase as prognostic markers in patients with advanced pNET treated with everolimus. J Clin Endocrinol Metab. 2011 Dec;96(12):3741-9.

18 Yamaguchi T, Machida N, Morizane C, Kasuga A, Takahashi H, Sudo K, et al. Multicenter retrospective analysis of systemic chemotherapy for advanced neuroendocrine carcinoma of the digestive system. Cancer Sci. 2014;105(9):1176-81.

19 Jacot W, Quantin X, Boher JM, Andre F, Moreau L, Gainet M, et al. Brain metastases at the time of presentation of non-small cell lung cancer: a multi-centric AERIO analysis of prognostic factors. Br J Cancer. 2001 Apr 6; 84(7):903-9.

20 Holdenrieder S, von Pawel J, Dankelmann E, Duell T, Faderl B, Markus A, et al. Nucleosomes, ProGRP, NSE, CYFRA 21-1, and CEA in monitoring first-line chemotherapy of small cell lung cancer. Clin Cancer Res. 2008 Dec 1;14(23):7813-21. 
21 Fan L, Wang Y, Chi C, Pan J, Xun S, Xin Z, et al. Chromogranin $\mathrm{A}$ and neurone-specific enolase variations during the first 3 months of abiraterone therapy predict outcomes in patients with metastatic castration-resistant prostate cancer. BJU Int. 2017 Aug; 120(2): 226-32.

22 Naito A, Taguchi S, Nakagawa T, Matsumoto A, Nagase Y, Tabata M, et al. Prognostic significance of serum neuron-specific enolase in small cell carcinoma of the urinary bladder. World J Urol. 2017 Jan;35(1):97-103.

23 Kanakis G, Kaltsas G. Biochemical markers for gastroenteropancreatic neuroendocrine tumours (GEP-NETs). Best Pract Res Clin Gastroenterol. 2012 Dec;26(6):791-802.

24 Huang L, Zhou JG, Yao WX, Tian X, Lv SP, Zhang TY, et al. Systematic review and metaanalysis of the efficacy of serum neuron-specific enolase for early small cell lung cancer screening. Oncotarget. 2017 Sep 8;8(38): 64358-72.

25 van Veenendaal LM, Bertolli E, Korse CM, Klop WMC, Tesselaar MET, van Akkooi ACJ. The clinical utility of neuron-specific enolase (NSE) serum levels as a biomarker for merkel cell carcinoma (MCC). Ann Surg Oncol. 2021 Feb;28(2):1019-28.

26 Xie J, Zhao Y, Zhou Y, He Q, Hao H, Qiu X, et al. Predictive value of combined preoperative carcinoembryonic antigen level and $\mathrm{Ki}$ 67 index in patients with gastric neuroendocrine carcinoma after radical surgery. Front Oncol. 2021;11:533039.

27 Zhuge X, Guo C, Chen Y, Feng L, Jia R, Zhao $Y$, et al. The levels of tumor markers in pancreatic neuroendocrine carcinoma and their values in differentiation between pancreatic neuroendocrine carcinoma and pancreatic ductal adenocarcinoma. Pancreas. 2018 Nov; 47(10):1290-5.

28 Konishi T, Shimada Y, Hsu M, Tufts L, Jimenez-Rodriguez R, Cercek A, et al. Association of preoperative and postoperative serum carcinoembryonic antigen and colon cancer outcome. JAMA Oncol. 2018 Mar; 4(3):309-15.

29 Luo G, Jin K, Cheng H, Liu C, Guo M, Lu Y, et al. Carbohydrate antigen 19-9 as a prognostic biomarker in pancreatic neuroendocrine tumors. Oncol Lett. 2017 Dec;14(6):6795800.

30 Isgrò MA, Bottoni P, Scatena R. Neuron-specific enolase as a biomarker: biochemical and clinical aspects. Adv Exp Med Biol. 2015;867: $125-43$.
31 van Adrichem RC, Kamp K, Vandamme T, Peeters M, Feelders RA, de Herder WW. Serum neuron-specific enolase level is an independent predictor of overall survival in patients with gastroenteropancreatic neuroendocrine tumors. Ann Oncol. 2016 Apr;27(4): 746-7.

32 Lokich JJ. Plasma CEA levels in small cell lung cancer. Correlation with stage, distribution of metastases, and survival. Cancer. 1982 Nov 15;50(10):2154-6.

33 Zhu H, Guo H, Li M, Zhang Y, Han A, Shi F, et al. Increased serum carcinoembryonic antigen level can predict poor survival of patients with small cell lung cancer. Transl Res. 2015 Oct;166(4):355-65.

34 Yang X, Wang D, Yang Z, Qing Y, Zhang Z, Wang $\mathrm{G}$, et al. CEA is an independent prognostic indicator that is associated with reduced survival and liver metastases in SCLC. Cell Biochem Biophys. 2011 Mar;59(2):1139.

35 Li Y, Bi X, Zhao J, Huang Z, Zhou J, Li Z, et al. CEA level, radical surgery, CD56 and CgA expression are prognostic factors for patients with locoregional gastrin-independent GNET. Medicine. 2016 May;95(18):e3567.

36 Zhao WX, Luo JF. Serum neuron-specific enolase levels were associated with the prognosis of small cell lung cancer: a meta-analysis. Tumour Biol. 2013 Oct;34(5):3245-8.

37 Huang Z, Xu D, Zhang F, Ying Y, Song L. Progastrin-releasing peptide and neuron-specific enolase: useful predictors of response to chemotherapy and survival in patients with small cell lung cancer. Clin Transl Oncol. 2016 Oct; 18(10):1019-25.

38 Zhou M, Wang Z, Yao Y, Zhou H, Liu M, Sun J. Neuron-specific enolase and response to initial therapy are important prognostic factors in patients with small cell lung cancer. Clin Transl Oncol. 2017 Jul;19(7):865-73.

39 Korse CM, Taal BG, Vincent A, Van Velthuysen ML, Baas P, Buning-Kager JC, et al. Choice of tumour markers in patients with neuroendocrine tumours is dependent on the histological grade. A marker study of Chromogranin A, neuron specific enolase, progastrin-releasing peptide and cytokeratin fragments. Eur J Cancer. 2012;48(5):662-71.

40 Shi M, Zhao W, Zhou F, Chen H, Tang L, Su B, et al. Neutrophil or platelet-to-lymphocyte ratios in blood are associated with poor prognosis of pulmonary large cell neuroendocrine carcinoma. Transl Lung Cancer Res. 2020 Feb;9(1):45-54.

41 Elisei R, Lorusso L, Piaggi P, Torregrossa L, Pellegrini G, Molinaro E, et al. Elevated level of serum carbohydrate antigen 19.9 as predictor of mortality in patients with advanced medullary thyroid cancer. Eur J Endocrinol. 2015 Sep;173(3):297-304
42 Li J, Luo G, Fu D, Jin C, Hao S, Yang F, et al. Preoperative diagnosis of nonfunctioning pancreatic neuroendocrine tumors. Med Oncol. 2011 Dec;28(4):1027-31.

43 Jin K, Xu J, Chen J, Chen M, Chen R, Chen Y, et al. Surgical management for non-functional pancreatic neuroendocrine neoplasms with synchronous liver metastasis: a consensus from the Chinese study group for neuroendocrine tumors (CSNET). Int J Oncol. 2016; 49(5):1991-2000.

44 Milione M, Maisonneuve P, Spada F, Pellegrinelli A, Spaggiari P, Albarello L, et al. The clinicopathologic heterogeneity of grade 3 gastroenteropancreatic neuroendocrine neoplasms: morphological differentiation and proliferation identify different prognostic categories. Neuroendocrinology. 2017; 104(1):85-93.

45 Lv Y, Han X, Zhang C, Fang Y, Pu N, Ji Y, et al. Combined test of serum CgA and NSE improved the power of prognosis prediction of NF-pNETs. Endocr Connect. 2018 Jan;7(1): 169-78.

46 Zheng Z, Chen C, Jiang L, Zhou X, Dai X, Song $Y$, et al. Incidence and risk factors of gastrointestinal neuroendocrine neoplasm metastasis in liver, lung, bone, and brain: a population-based study. Cancer Med. 2019 Dec; 8(17):7288-98.

47 Pavel M, O’Toole D, Costa F, Capdevila J, Gross D, Kianmanesh R, et al. ENETS consensus guidelines update for the management of distant metastatic disease of intestinal, pancreatic, bronchial neuroendocrine neoplasms (NEN) and NEN of unknown primary site. Neuroendocrinol. 2016;103(2):172-85.

48 Ilett EE, Langer SW, Olsen IH, Federspiel B, Kjær A, Knigge U. Neuroendocrine carcinomas of the gastroenteropancreatic system: a comprehensive review. Diagnostics. 2015 Jun; 5(2):119-76.

49 Romeo P, Colombo C, Granata R, Calareso G, Gualeni AV, Dugo M, et al. Circulating miR375 as a novel prognostic marker for metastatic medullary thyroid cancer patients. Endocr Relat Cancer. 2018 Mar;25(3):217-31.

50 Oversoe SK, Sorensen BS, Tabaksblat EM, Gronbaek H, Kelsen J. Cell-Free DNA and clinical characteristics in patients with small intestinal or pancreatic neuroendocrine tumors. Neuroendocrinol. 2021.

51 Bocchini M, Nicolini F, Severi S, Bongiovanni A, Ibrahim T, Simonetti G, et al. Biomarkers for pancreatic neuroendocrine neoplasms (PanNENs) management-an updated review. Front Oncol. 2020 May 27;10:831.

52 Lewis MA, Yao JC. Molecular pathology and genetics of gastrointestinal neuroendocrine tumours. Curr Opin Endocrinol Diabetes Obes. 2014 Feb;21(1):22-7. 